\title{
Progenitors of type Ia supernovae and their surviving companion stars
}

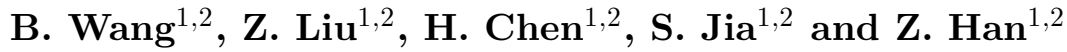 \\ ${ }^{1}$ Yunnan Observatory, CAS, Kunming, 650011, China (email: wangbo@ynao.ac.cn) \\ ${ }^{2}$ Key Laboratory for the Structure and Evolution of Celestial Objects, CAS, Kunming, \\ 650011, China
}

\begin{abstract}
Type Ia supernovae (SNe Ia) are the best cosmological distance indicator, however, there is still no agreement on the nature of their progenitors. There are mainly two progenitor scenarios, i.e., the single degenerate (SD) and double degenerate (DD) scenarios. In this article, we introduced our binary evolution models of various progenitor scenarios. We also obtained many properties of the surviving companions from various SD systems, which may be helpful for identifying SN Ia progenitor model. At present, it seems likely that more than one progenitor model may be required to explain the observed diversity of SNe Ia.
\end{abstract}

Keywords. Binaries: close - supernovae: general - white dwarfs

\section{Introduction}

Type Ia supernovae (SNe Ia) play an important role in astrophysics, especially in the study of cosmic evolution. However, the nature of their progenitors is still unclear, raising doubts about the calibration that is purely empirical and based on nearby SN Ia sample. SNe Ia are believed to be thermonuclear explosions of carbon-oxygen white dwarfs (CO WDs). Observational characteristics of SNe Ia imply that the SN explosion occurs when a CO WD reaches the Chandrasekhar $(\mathrm{Ch})$ limit. There are mainly two scenarios to produce the $\mathrm{Ch}$ mass $\mathrm{CO}$ WDs: the single degenerate (SD) scenario, where a CO WD accretes material from a non-degenerate companion (Hachisu et al. 1996; Li \& van den Heuvel 1997; Han \& Podsiadlowski 2004), and the double degenerate (DD) scenario, where two CO WDs with a total mass larger than the Ch limit coalesce (Webbink \& Iben 1987; Han 1998). However, it is quite likely that the merger product experiences a core collapse rather than a thermonuclear explosion. For a recent comprehensive review on this subject see Wang \& Han (2012).

\section{Progenitor models and surviving companions}

In the observations, there are 3 subclasses of SNe Ia based on their time delay (from star formation to SN explosion), short time delay SNe Ia with a time delay less than $0.1 \mathrm{Gyr}$, intermediate time delay SNe Ia with a time delay between 0.1 and $1 \mathrm{Gyr}$, and long time delay SNe Ia with a time delay larger than 1 Gyr. Theoretically, the SD scenario has three channels to produce $\mathrm{SNe} \mathrm{Ia}$, i.e., the $\mathrm{WD}+\mathrm{He}$ star, $\mathrm{WD}+\mathrm{MS}$ and $\mathrm{WD}+\mathrm{RG}$ channels. In the WD+He star channel, a CO WD accretes material from a helium star to increase its mass to the $\mathrm{Ch}$ limit. This channel is used to explain short time delay SNe Ia, since $\mathrm{CO}$ WD+He star systems are produced from the evolution of intermediate mass binaries. In the WD+MS channel, a WD accretes matter from a main sequence (MS) star or a star slightly evolved away from the MS, and this channel is used to explain intermediate time delay $\mathrm{SNe}$ Ia. In the $\mathrm{WD}+\mathrm{RG}$ channel, the mass donor is a low mass 
red giant $(\mathrm{RG})$, and this channel is responsible for long time delay SNe Ia. The DD scenario may produce both short time delay SNe Ia and long time delay ones. For details see Fig. 2 of Wang et al. (2010b).

In order to calculate SN Ia birthrates from various channels, we first employed Eggleton's stellar evolution code (Eggleton 1971; Han et al. 1994) and adopted the prescription of Hachisu et al. (1996) for the mass-accretion efficiency of a CO WD, and carried out detailed binary evolution calculations for close CO WD binaries, and mapped out the initial parameters in the orbital period-secondary mass plane (for a range of WD masses) which lead to an SN Ia. We then implemented these results into a binary population synthesis study, which is to follow the evolution of millions of primordial binaries, to obtain SN Ia birthrates. For details of the calculations, we refer the reader to Han (1998) for the DD scenario, to Han \& Podsiadlowski (2004, 2006) and Wang et al. (2010a) for the WD+MS channel, Wang et al. (2009a,b) for the WD+He star channel, Wang \& Han (2010a) for the WD+He star channel with various metallicities, Wang \& Han (2010b) for the WD+RG channel.

The companion in the SD scenario would survive after the SN explosion and potentially be identifiable, whereas an explosion following the merger of two WDs would leave no remnant (Han 2008). We obtained many properties of the surviving companions from various SD systems, which may be helpful for identifying SN Ia progenitor model. The SD scenario can also help to understand the formation of hypervelocity stars (Wang \& Han 2009) and single low mass helium WDs (Justham et al. 2009; Wang \& Han 2010c).

\section{Conclusions}

A promising method to test SN Ia progenitor models is to search their surviving companions. At present, it seems likely that more than one progenitor model may be required to explain the observed diversity of SNe Ia.

This work is supported by the Natural Science Foundation of China under grant nos. 11033008, 11103072, 2009CB824800 and KJCX2-YW-T24.

\section{References}

Eggleton, P. P. 1971, MNRAS, 151, 351

Hachisu, I., Kato, M., \& Nomoto, K. 1996, ApJ, 470, L97

Han, Z. 1998, MNRAS, 296, 1019

Han, Z. 2008, ApJ, 677, L109

Han, Z. \& Podsiadlowski, P. 2004, MNRAS, 350, 1301

Han, Z. \& Podsiadlowski, P. 2006, MNRAS, 368, 1095

Han, Z., Podsiadlowski, P., \& Eggleton, P. P. 1994, MNRAS, 270, 121

Justham, S., Wolf, C., Podsiadlowski, P., \& Han, Z. 2009, A $E A$ A, 493, 1081

Li, X.-D. \& van den Heuvel, E. P. J. 1997, A\&A, 322, L9

Wang, B. \& Han, Z. 2009, A\& $A$, 508, L27

Wang, B. \& Han, Z. 2010a, A\&A, 515, A88

Wang, B. \& Han, Z. 2010b, RAA (Res. Astron. Astrophys.), 10, 235

Wang, B. \& Han, Z. 2010c, MNRAS, 404, L84

Wang, B. \& Han, Z. 2012, New Astron. Rev., 56, 122

Wang, B., Li, X.-D., \& Han, Z. 2010a, MNRAS, 401, 2729

Wang, B., Liu, Z., Han, Y., et al. 2010b, ScChG (Sci. China Ser. G), 53, 586

Wang, B., Meng, X., Chen, X., \& Han, Z. 2009a, MNRAS, 395, 847

Wang, B., Chen, X., Meng, X., \& Han, Z. 2009b, ApJ, 701, 1540

Webbink, R. F. 1984, ApJ, 277, 355 